\title{
SSAAR: Enhanced Spatial Reusability for Optimal Path Selection in Wireless Sensor Networks
}

\author{
R. Deepthi, D. Sai Eswari, Afreen Rafiq and K. Srinivas \\ Department of Computer Science and Engineering, Sri Satya Sai University of Technology and Medical Sciences, \\ Sehore-466001, Madhya Pradesh, India; deepthideepu66@gmail.com, saieswari3@gmail.com, \\ afreenrafiq2@gmail.com, katakamsrinivas@gmail.com
}

\begin{abstract}
The advancements made in the wireless sensor networks have enabled the research communities to delve into the study of spatial reusability concepts. Due to the restricted capacity in wireless links, it is a significant to select the optimal route for packet transmission. The achievement of optimal route selection is verified by the high end-to-end throughput rate. Routing in WSNs is very challenging due to the inherent characteristics that distinguish these networks from other wireless networks like mobile ad hoc networks or cellular networks. In this paper, we have proposed an improved spatial reusability process using Single Path and Anypath Routing Protocols (SSAAR). The task of single path routing is to minimize the cost of the nodes in wireless links, whereas the task of any path routing is to assists the intermediate node who overhears the packet to participate in packet forwarding. Experimental results have shown the efficacy of our proposed work in terms of cost minimization functionalities.
\end{abstract}

Keywords: Cost Minimization, Packet Forwarding, Single Path and Anypath Routing Protocols, Wireless Sensor Networks, Wireless Links

\section{Introduction}

Recently, the research on wireless technologies has developed a greater impact among the network users ${ }^{1}$. Wireless networks is an emerging technology in which users can make use of its from anywhere at anytime. The study towards wireless networks have enabled the researchers by its functionalities like fault tolerance, self-configuration and scalability. The research over multi-hop wireless networks consists of voluminous amount of wireless devices that covers mesh networks and mesh gateways 2 . Thus, a phenomenal growth has been seen in the development of wireless devices and networks.

Energy availability and reliability are the most prominent issues faced in the multi-hop wireless sensor networks. The real-time applications deployed using multi-path routing systems demands optimal reliability and the quality of service. In order to achieve better reliability, the availability of energy among the nodes is a significant factor. Hence, reliability is directly proportional to the energy availability. The task of multipath routing is to get hold of the destination using single path or any path routing systems. In case of any misbehavior occur in node, and then the re-routing process is initiated ${ }^{3}$. Relatively, each node cooperates with other nodes to form a path and behaves like relay for transmitting the packets. The instability of the topology link or node failures could results in disconnected routes.

In fact, the paths are formed without any knowledge about the nodes ${ }^{4}$. Thus, the presence of failure nodes can't be detected in an untimely phase. The transformation of packets between failure nodes can affect the performance of the networks. Similarly, the concept of multipath scheme is to supply the energy without any interference at the same time. There are two paths, namely, primary paths and protection paths. Both these paths will not synchronize with other nodes while transmitting the packets. To resolve this, the concept of network interference is

*Author for correspondence 
achieved. Since the multipath routing inquires more than one route to process the data. Henceforth, Route searching is a vital task in multipath routing scheme. Routing in wireless networks operates based on the received requests in both dynamic and static routing. The two paths, viz, primary path and protection path are used for routing analysis. Primary path deals with failure nodes whereas protection path deals with reserved request $t^{-}$.

The shared nodes could affect the network performance, if any failure node is detected. It effectively assists to save the energy. The ability of multipath routing schemes in providing a better QoS in transferring multimedia applications such as voice, video and data, has been proved in a number of previous studies, such as in ${ }^{\underline{6}}$. For every received request, it performs two paths for processing the data. If some network criteria are satisfied, it is possible to use a same link to protect multiple primary paths. Reusability of a protection link is the ability to protect the multiple paths. In wireless mesh networks, this work discuss about the reusability ${ }^{?}$. The received user's request is further reused for energy consumption in the networks.

The rest of the paper is organized as follows: Section II depicts the related work; Section III describes the proposed work; Section IV describes the experimental analysis and concludes in Section V.

\section{Literature Survey}

This section depicts the related works carried out by other researchers. The author in ${ }^{10}$ studied about the video streaming process that specifically deals with bandwidth and energy constrained analysis. In this single video frame is subdivided into different frames and then each frame is transmitted to its sub-stream process. It is deployed over Directional Geographical Routing (DGR) which studied about the parameters load balance and bandwidth. The author in 11 studied about the multipath routing scheme which devised that Quality of Service in the perspective of bandwidth, hop count and end-to- end delay in MANET environments. The primary path may get disrupted when the route discovery processes are properly initiated. A new path is designed for incessant data transmission systems. And thus, they achieved high reliability and low overloaded systems.

Similarly, the author in $\underline{12}$ studied the performance of the network under diverse path routing in order to enhance the packets delivery ratio, reliability and maximizing the effect of multipath routing design. The author in $\frac{13}{3}$ pre- sented an end-to-end throughput rate using multipath routing process with less inference among the networks. The author in ${ }^{\underline{14}}$ presented about the node disjoint paths in indoor environments in terms of route discovery and message control overhead. Relatively, an enhancement of end-to-end reliability process is studied by author in ${ }^{15}$. A copy of data is being sent to the different paths and also the diversity in frequency is manipulated in multi-radio environment.

Similarly, the author in $\frac{16}{16}$ studied about the multipath routing that helps to increase the throughput rate. Another study in ${ }^{17}$ for detecting and resolving for dynamic path deterioration in wireless networks, they presented interference-aware multi-radio routing protocol. When radical link deterioration happens, this proposed protocol dynamically reconstructs a source initiated path. Another approach for the multipath design was studied in ${ }^{1 \frac{18}{}}$. In wireless ad-hoc network, they studied the problem of finding the minimum energy disjoint paths. They have concentrated in static ad-hoc networks. They used all nodes along the primary paths named common nodes after finding a primary path in each request. To form disjoint paths, they shared those common nodes to find another path.

The author in $\frac{19}{19}$ proposed a multipath routing protocol named AODV-DM that assisted to find multiple paths with less interference. An insulating region is formed around the primary path after finding a primary path. It contains all the edges in the primary path within the interference range of each node ${ }^{20}$. To reduce the potential network interference with the found primary path, a protection path must be selected and established outside the insulating region. By the use of insulating region, most of the network links would be eliminated. From the reviews, the challenges in spatial reusability analysis are:

- Restricted wireless communication media which involve optimal route selection process in order to enhance the end-to-end throughput.

- The rate of data transmission is higher in case of delivering single packets.

\section{Proposed Work}

\subsection{Motivation}

The advancements made in wireless networks have enabled the wireless users to adopt the wireless services. In order to achieve best data transmission process, the selection of optimal route between sender and receiver 
node is a vital thing. Though, the prior routing protocols exhibits a better route selection, but it ended up with the issue of high end-to-end throughput. End to End throughput is the amount of data travelled from sender node to the receiver node within stipulated period of time. To overcome from this issue, the spatial reusability concept has been explored by several researchers. Most of the wireless networks environment suffers from the spatial wastage towards the communication process. This motivates us to delve into the study of spatial reusability in multi-hop wireless networks environment.

\subsection{Devised Spatial Aware Path Routing Protocols}

This section depicts the general workflow of our proposed spatial aware path routing protocols. This is achieved by two protocols, namely Singe path routing protocols and Anypath routing protocols. The proposed algorithm is explained as follows:

\subsubsection{System Model}

Let us consider a set of static $\mathrm{N}$ nodes which are connected via multihop wireless networks with fixed transmission rate. The deployment of single path routing protocols incurs Expected Transmission Count Metric (ETX) that states probability transmission rate from node $i$ to node $i$. Thus, the probability of transmitting the packets from node $i$ to node $j$ is given as:

$$
\mathrm{z}_{i}=\frac{1}{p_{i j-p j i}}
$$

Let $\mathrm{T}_{\text {data }}$ represents the transmission time rate of packet and $\mathrm{T}_{\text {ack }}$ represents the packet acknowledgment. Henceforth, the expected time for packet delivery from node $i$ to node $j$ is given as:

$$
t_{i j}=Z_{i} * T_{\text {data }}+Z_{i} * p_{i j-T_{a c k}}
$$

Similarly, anypath routing calculated from the set of forwarder nodes $F_{i}$. With the use of forwarder nodes, the expected number of transmissions by node $\mathrm{i}$ is given as:

$$
\mathrm{z}_{i F_{\mathrm{i}}}=\frac{1}{1-\pi_{j \in F_{\mathrm{i}}}\left(1-p_{i j}\right)}
$$

Based on the propagation medium, the two distant wireless links can work if they spatial distant from each other. Let us explain about the working of Single path routing and Anypath routing protocols.

\subsubsection{Spatial Reusability Aware -Single Path Routing Protocols (SR-SPRP)}

The task of the Single path routing protocol is to select the optimal paths that yields better performance at lower cost. The proposed steps involved in Single Path Routing Protocols are:

1. Input: Path $\mathrm{p}$, Link cost $\left(\mathrm{t}_{i, j}\right) € \mathrm{p}$, Link graph $\mathrm{G}=(\mathrm{P}, \mathrm{E})$.

2. In accord to decreasing order, the links in set $\mathrm{P}$ are arranged.

3. Estimating the Link delivery time for the set I (collection of non-interfering paths) of wireless links as:

$$
c(I)=\max \left\{t_{1} i j \mid(i, j) € I\right\}
$$

4. For every set I, the spatial resuability aware path delivery time is given as:

$$
C=\sum_{i \in I} c(I)
$$

5. Consider each path as conflict graph $\mathrm{G}=(\mathrm{V}, \mathrm{E})$ where $\mathrm{V}$ is the links and $\mathrm{E}$ is the interferences.

6. Thus, every path $\mathrm{P}$ in the non-interfering set $\mathrm{I}$, we compute $\mathrm{G}$ where $\mathrm{E}=\left\{(\mathrm{i}, \mathrm{j}),\left(\mathrm{i}^{\prime}, \mathrm{j}^{\prime}\right)\right\}$ that holds interference among the links.

7. Output: Optimal non-interfering paths with its optimal costs.

\subsubsection{Spatial Reusability Aware- Anypath Routing Protocols (SR-APRP):}

The role of anypath routing protocols is to pick the set of source, intermediate and destination nodes, so as to achieve better packet delivery with cost minimized factor. The proposed steps involved in anypath routing protocols are:

1. Input: Network Graph $\mathrm{G}=(\mathrm{N}, \mathrm{E})$, source node and destination node.

2. Estimating the distance cost $\vec{C}=\left(C_{i}\right) i \varepsilon Q$ with forwarder lists $\vec{F}=\left(F_{i}\right) i \epsilon Q$.

3. When the node cost is lower, it implies that the destination node is at reachable position.

4. Using recursive function, the probability between node $i$ and node $j$ is given as:

1 if $j=i$; otherwise $\sum_{k \in F_{\mathrm{i}}} \omega_{i k} * \varphi(k, j)$ if $j \varepsilon Q_{j}^{c}<C_{i}$

5. Thus, the packet delivery from source node $i$ to the destination node $\mathrm{j}$ is given as:

$$
t_{F_{\mathrm{i}}}=\varphi(s r c, i) * t_{i} F_{i}
$$


6. The total cost of packet delivering from source to destination node is:

$$
C_{s r c}=\sum_{i \in I} c(I)
$$

7. Output: A set of participating nodes $\mathrm{Q}$, and the corresponding profile of $\operatorname{cost} \mathrm{C}$ and forwarder lists $\mathrm{F}$.

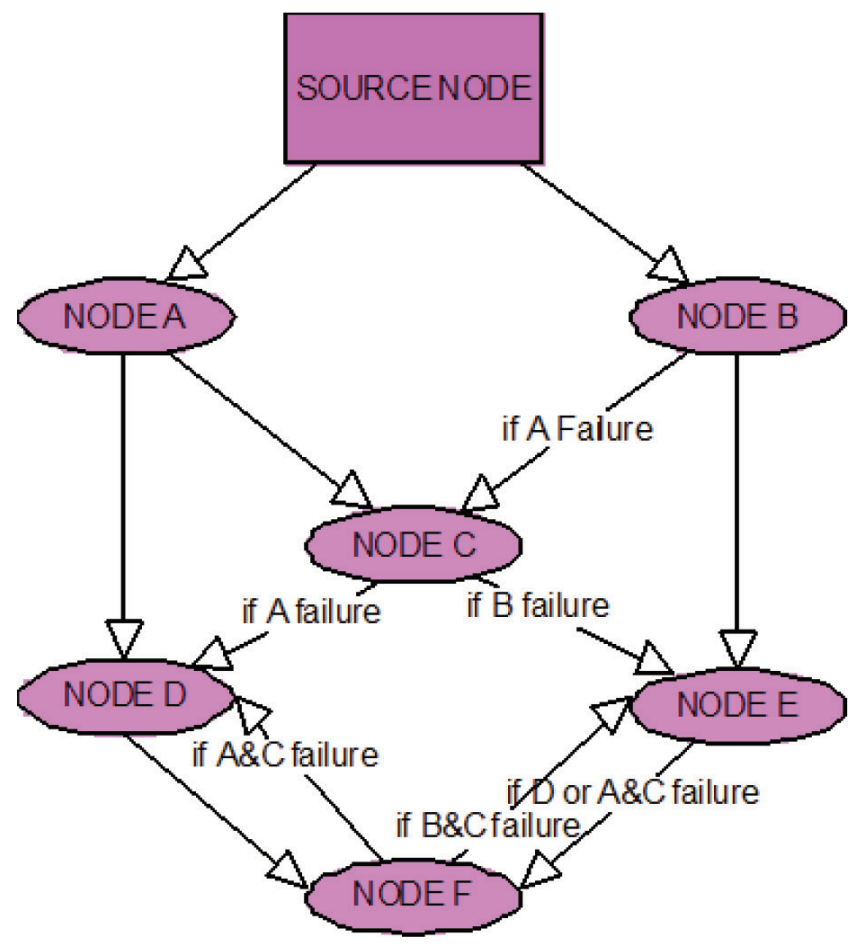

Figure 1. Proposed workflow.

\section{Experimental Analysis}

This section depicts simulation analysis of our proposed routing protocols. The Table 1 depicts the simulation parameters used in our network environment. To be detailed, we randomly picked 200 source-destination pairs for single-path and anypath routing, respectively, from those that result in different routing decisions from the compared routing algorithms for clarity. The throughput of each source-destination pair was averaged over 100 runs, each of which lasted 10 minutes.

Table 1. Simulation parameters

\begin{tabular}{|l|l|}
\hline Parameter & Value \\
\hline No.of nodes & 100 \\
\hline Area & $2000 \mathrm{~m} * 2000 \mathrm{~m}$ \\
\hline
\end{tabular}

\begin{tabular}{|l|l|}
\hline RTS/CTS & Off \\
\hline Transmission rate & $11 \mathrm{Mbps}$ \\
\hline Traffic Generator & CBR \\
\hline CBR rate & $5 \mathrm{Mbps}$ \\
\hline Packet size & 1500 bytes \\
\hline
\end{tabular}

With the defined set of nodes, the numbers of edges get decreased when the area size increases. Consider instances 303 requests are satisfied under the area of $1000 \mathrm{~m} * 1000 \mathrm{~m}$ and 53 requests are satisfied under the area of $2500 * 2500 \mathrm{~m}$. The obtained results are further compared with the outcomes of SAAR and AODVDM schemes. The results depicts that AODVDM incurs high time in insulating region and the SAAR schemes works incurs high time in multi path routing systems shown in Figure 2.

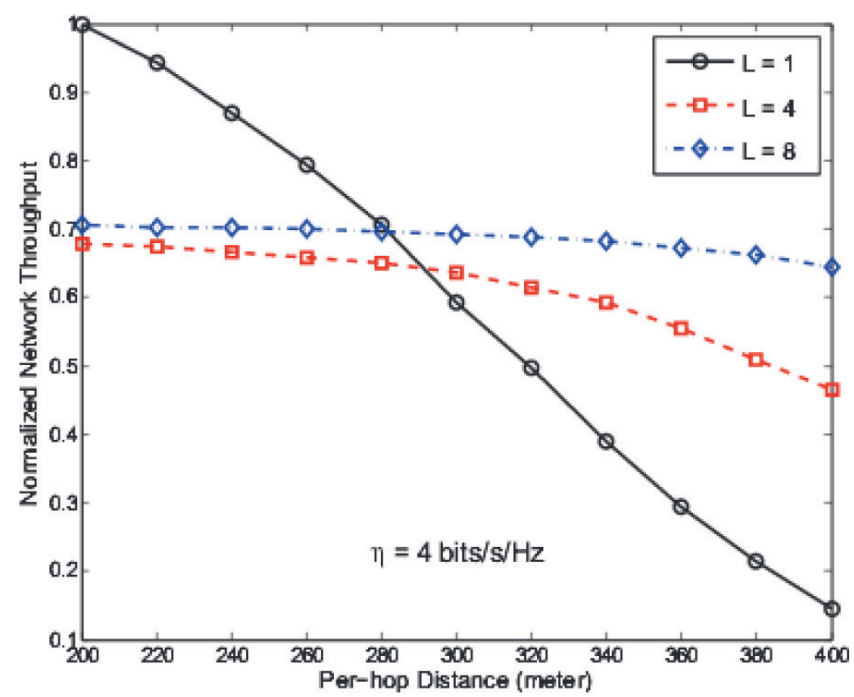

Figure 2. Normalized network throughput in multi-hop distance.

The proposed SSAAR model satisfies more request than in SAR and AODVDM by considering interference and protection links reusability. It is inferred from Figure 3 that AODV-DM can satisfy less number of requests because it hide all the edges that interfere with the primary or the protection path and it can also hide most of the links in the networks. By hiding more number of interfered edges in the graph, AODV-DM scheme will lead to a drop in the satisfied ratio and also increasing the number of nodes within the same area size. 


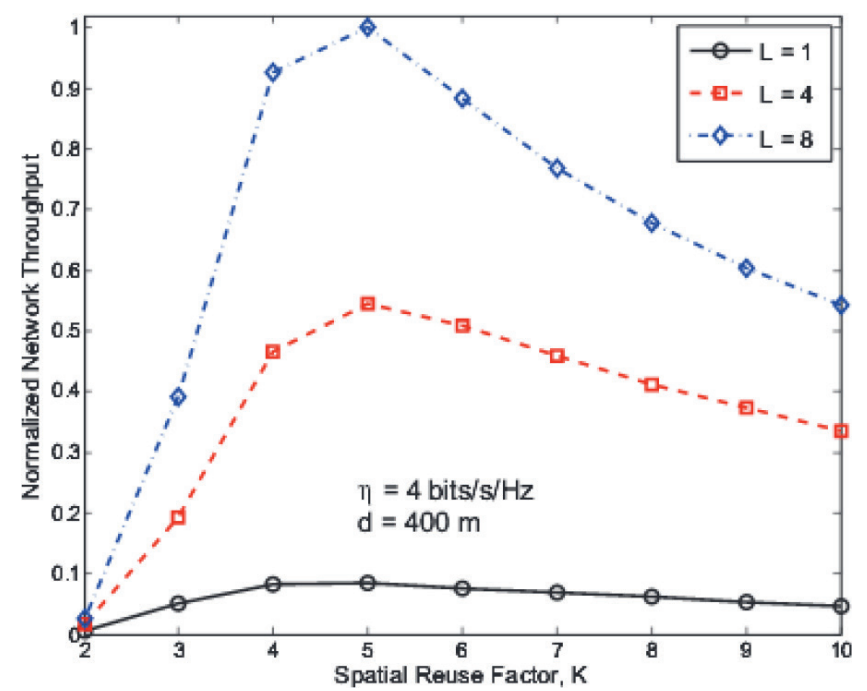

Figure 3. Normalized network throughput using spatial reuse factor.

\section{Conclusion}

The technology development towards wireless sensor networks has seen phenomena growth which attracts variety of wireless users. Routing is the significant task due to the inherent characteristics that distinguish these networks from other wireless networks. The wireless networks services are restricted in many ways. Spatial reusability is the concept introduced to overcome the issue of optimal path selection. Thus, we have proposed an improved spatial reusability process using single path and anypath routing protocols (SSAAR). The task of single path routing is to minimize the cost of the nodes in wireless links, whereas the task of any path routing is to assists the intermediate node who overhears the packet to participate in packet forwarding. Experimental results have shown the efficacy of our proposed work in terms of cost minimization functionalities. The proposed SSAAR technique is validated via simulating the packets and evaluating with throughput rate and spatial reuse factor in pair-wise. The similar computation model becomes significant in larger networks. And better performance acheived in 2-hop interference than the 1-hop interference.

\section{References}

1. Tong Meng et. al. Spatial reusability-aware routing in multihop wireless networks, IEEE Transactions on Computers. 2016; 65(1). Crossref.
2. Adya A., Bahl P., Padhye J., Wolman A., Zhou L. A multi radio unification protocol for IEEE 802.11 wireless networks, Proc. 1st Int. Conf. Broadband Netw. 2004; p. 344-54. Crossref.

3. Broch J., Maltz D.A., Johnson D.B., Hu Y.-C., Jetcheva J.G. A performance comparison of multi-hop wireless ad hoc network routing protocols, Proc. 4th Annu. ACM/IEEE Int. Conf. Mobile Comput. Netw. 1998, p. 85-97.

4. Chachulski S., Jennings M., Katti S., Katabi D. Trading structure for randomness in wireless opportunistic routing, Proc. SIGCOMM Conf. Appl., Technol., Archit. Protocols Comput. Commun., 2007.

5. Johnson D.B., Maltz D.A. Dynamic source routing in adhoc wireless networks, Mobile Comput., 1992; 353:153-81.

6. Perkins C.E., Bhagwat P. Highly dynamic Destination Sequenced Distance-Vector (DSDV) routing for mobile computers, Proc. Conf. Commun. Archit., Protocols Appl., 1994, p. 234-44.

7. Laufer R.P., Dubois-Ferriere H., Kleinrock L. Multirateanypath routing in wireless mesh networks, Proc. INFOCOM, 2009, p. 37-45.

8. Lin Y., Li B., Liang B. Codeor: Opportunistic routing in wireless mesh networks with segmented network coding, Proc. IEEE Int. Conf. Netw. Protocols, 2008, p. 13-22.

9. Kim T.S., Hou J.C., Lim H. Improving spatial reuse through tuning transmits power, carrier sense threshold, and data rate in multihop wireless networks, Proc. 12th Annu. Int. Conf. Mobile Comput. Netw., 2006, p. 366-77. Crossref.

10. Draves R., Padhye J., Zill B. Routing in multi-radio, multihop wireless mesh networks, Proc. 10th Annu. Int. Conf. Mobile Comput. Netw., 2004, p. 114-28.

11. Jones N.M., Shrader B., Modiano E. Optimal routing and scheduling for a simple network coding scheme, Proc. INFOCOM, 2012, p. 352-60. Crossref.

12. Kim T.-S., Hou J.C., Lim H. Improving spatial reuse through tuning transmit power, carrier sense threshold, and data rate in multihop wireless networks, MOBICOM, 2006.

13. Laufer R.P., Dubois-Ferri'ere H., Kleinrock L. Multirate anypath routing in wireless mesh networks, INFOCOM, 2009.

14. Lin Y., Li B., Liang B. Codeor: Opportunistic routing in wireless mesh networks with segmented network coding, ICNP, 2008.

15. Padhye J., Agarwal S. Padmanabhan V.N., Qiu L., Rao A., Zill B. Estimation of link interference in static multi-hop wireless networks, Internet Measurment Conference, 2005. Crossref.

16. Pan M., Zhang C., Li P., Fang Y. Joint routing and link scheduling for cognitive radio networks under uncertain spectrum supply, INFOCOM, 2011.

17. Perkins C.E., Belding-Royer E.M. Ad-hoc on-demand distance vector routing, WMCSA, 1999. 
18. Perkins C.E., Bhagwat P. Highly dynamic destinationsequenced distance-vector (DSDV) routing for mobile computers, SIGCOMM, 1994.

19. Radunovic B., Gkantsidis C., Key P.B., Rodriguez P. An optimization framework for opportunistic multipath routing in wireless mesh networks, INFOCOM, 2008.
20. Rahul H., Hassanieh H., Katabi D. Sourcesync: A distributed wireless architecture for exploiting sender diversity, SIG-COMM, 2010. 